\title{
Upper Respiratory System
}

National Cancer Institute

\section{Source}

National Cancer Institute. Upper Respiratory System. NCI Thesaurus. Code C33839.

The sinuses and those parts of the respiratory system above the trachea. It includes the nares, nasopharynx, oropharynx, larynx, vocal cords, glottis and upper trachea. 